\title{
A case of primary amenorrhea with 46,XY Karyotype: Androgen insensitivity syndrome (AIS)
}

\author{
Yamini Pokale $^{1,2}$, Ajinkya Jadhav, Bharat Kalthe ${ }^{2}$, Ushang Kate ${ }^{2}$, Prashant \\ Khadke $^{1}$ \\ 1 Department of Biotechnology, Jagdish Prasad Jhabarmal Tibrewala University, India \\ 2 Department of Cytogenetics, PreventiNe Life Care Pvt Ltd, India.
}

\begin{abstract}
We studied a proband of 23 years female, who had history of primary amenorrhea and was diagnosed with Partial androgen insensitivity syndrome (PAIS), an X-linked disorder caused by mutation in androgen receptor gene. Karyotyping was conducted by analysis of G-banded chromosomes using heparinized peripheral blood sample. The molecular study using PCR for confirmation of the presence of $Y$ chromosome (SRY gene) was performed. Based on the clinical findings, hormonal profile and USG report of the proband, it was confirmed that the proband is diagnosed with PAIS a type of AIS which was further counseled for the better future.
\end{abstract}

Keywords - primary amenorrhea, Androgen insensitivity syndrome, ambiguous genitalia, SRY gene, clitoromegaly, mutation, gynecomastia

\section{INTRODUCTION}

The The first medical report on Androgen insensitivity syndrome (AIS) was published in 1953 by J. M. Morris, an American gynaecologist and was initially described in 82 patients. The syndrome was designated "testicular feminization syndrome", because the testes produce hormones with estrogen-like actions [1]. AIS is caused by mutations in the Androgen Receptor (AR) gene. In 1989, the exact localization of the human AR gene was defined on Xq11-12 and in the same year the first proof that AIS was caused by mutations in the AR gene was published by Brown et al [2].

AIS present at several differentiation from genetic defects to end organ resistance thereby producing gender dilemma dispelled by sex hormones signature [3]. It is generally accepted that defects in the AR gene prevent the normal development of both internal and external genital structures in 46,XY individuals, causing a variety of phenotypes ranging from complaints of male infertility to completely normal female external genitalia [4]. It is an X linked disorder associated with vaginal and uterine agenesis in women with a 46,XY karyotype.

The estimated prevalence of AIS is between 1 in 20,000 and 1 in 99,000 genetic males [5]. Precise diagnosis of AIS requires clinical, hormonal and molecular investigation and is of great importance for appropriate gender assignment and management in general [4].

AIS have traditionally been classified into three clinical subgroups based on the genital phenotype [6]:

1. Complete Androgen Insensitivity syndrome (CAIS): Individuals with CAIS have normal female external genitalia. They typically present either before puberty with masses in the inguinal canal that are subsequently identified as testes or at puberty with primary amenorrhea and sparse to absent pubic or axillary hair. Sexual identity and orientation are unaffected.

2. Partial androgen insensitivity syndrome (PAIS): Individuals with PAIS are predominantly female, predominantly male, or ambiguous genitalia. However affected individuals have signs of external genital masculinization including clitoromegaly or posterior labial fusion.

3. Mild androgen insensitivity syndrome (MAIS) Individuals with MAIS having typical male genitalia. They usually present with gynecomastia at puberty. Spermatogenesis may or may not be impaired. In some instances, the only observed abnormality appears to be male infertility; therefore, MAIS could explain some idiopathic male infertility.

\section{MATERIALS AND METHODS}

The PreventiNe Life Care is the genetic research centre located at Navi Mumbai. We present the case of a 23 years old phenotypic female patient, who presented with primary amenorrhea. The proband was referred to our laboratory for karyotyping. Clinical examination revealed absence of secondary sex characteristics, complete absence of breast development. Her external genitalia were normal with absence of axillary and pubic hair. She was 5.8 feet tall, weighted $52 \mathrm{kgs}$. 
Peripheral blood $(2 \mathrm{ml})$ was collected in heparin vacutainer (Becton Dickinson, USA). Whole blood $(0.5 \mathrm{ml})$ culture was set up in 5ml RPMI 1640 media (GIBCO BRL, USA) containing fetal bovine serum and phytohemagglutinin using standard cytogenetic techniques. Cultures were harvested and Karyotyping was performed on G-bands produced with the trypsin and giemsa (GTG) - banded chromosome preparations. The chromosomal status was analyzed using Karyoimager software by Carl Zeiss. At least 25 metaphases were analyzed. All chromosomal abnormalities were reported in accordance with the current international standard nomenclature (ISCN)[7].

Hormonal evaluation is done from serum using Chemi Luminescent Immuno Assay (C.L.I.A)(Table-1)

\section{RESULTS}

Hormonal evaluation (Table-1) revealed serum FSH and serum LH were within the normal range for male while testosterone level was low as compare to the normal range for male. Clinical evaluation of serum testosterone, along with serum LH, assists in evaluation of hypogonadal males.

Ultrasound examination of pelvic organs revealed uterus small in size measuring $2.3 \times 0.7 \times 2.6 \mathrm{cms}$. Both ovaries were visualized and showed non dominant follicles within. The right ovary measures $2.0 \times 1.1 \times 1.4 \mathrm{cms}$ and left ovary measures $1.5 \times 1.2 \times 1.6 \mathrm{cms}$. The Ultrasound report showed Hypo-plastic uterus.

Conventional cytogenetic analysis from peripheral blood lymphocyte culture was done. The banding resolution of the metaphase chromosomes (ISCN) was 450-500. The metaphase spreads were scored and compared against idiogram. The karyotype of proband from peripheral blood lymphocytes was that of a normal male (46,XY) (Fig.1). Presence of Sex determining Region on Y chromosome (SRY) was confirmed by Polymerase Chain Reaction (PCR) by molecular study (Fig. 2).

IV. FIGURES AND TABLES

Table 1 - Hormone analysis results

\begin{tabular}{|c|c|c|c|}
\hline & Hormones & $\begin{array}{lll}\text { Nonge } & \text { male } \\
\end{array}$ & Proband \\
\hline & Testosterone & $280-800 \mathrm{ng} / \mathrm{dL}$ & $2.50 \mathrm{ng} / \mathrm{dL}$ \\
\hline$(\mathrm{FSH})$ & Follicle-stimulating hormone & $1.4-18.1 \mathrm{mlU} / \mathrm{ml}$ & $5.17 \mathrm{mlU} / \mathrm{ml}$ \\
\hline & Luteinizing hormone ( LH) & $1.5-9.3 \mathrm{mlU} / \mathrm{ml}$ & $3.98 \mathrm{mlU} / \mathrm{ml}$ \\
\hline
\end{tabular}

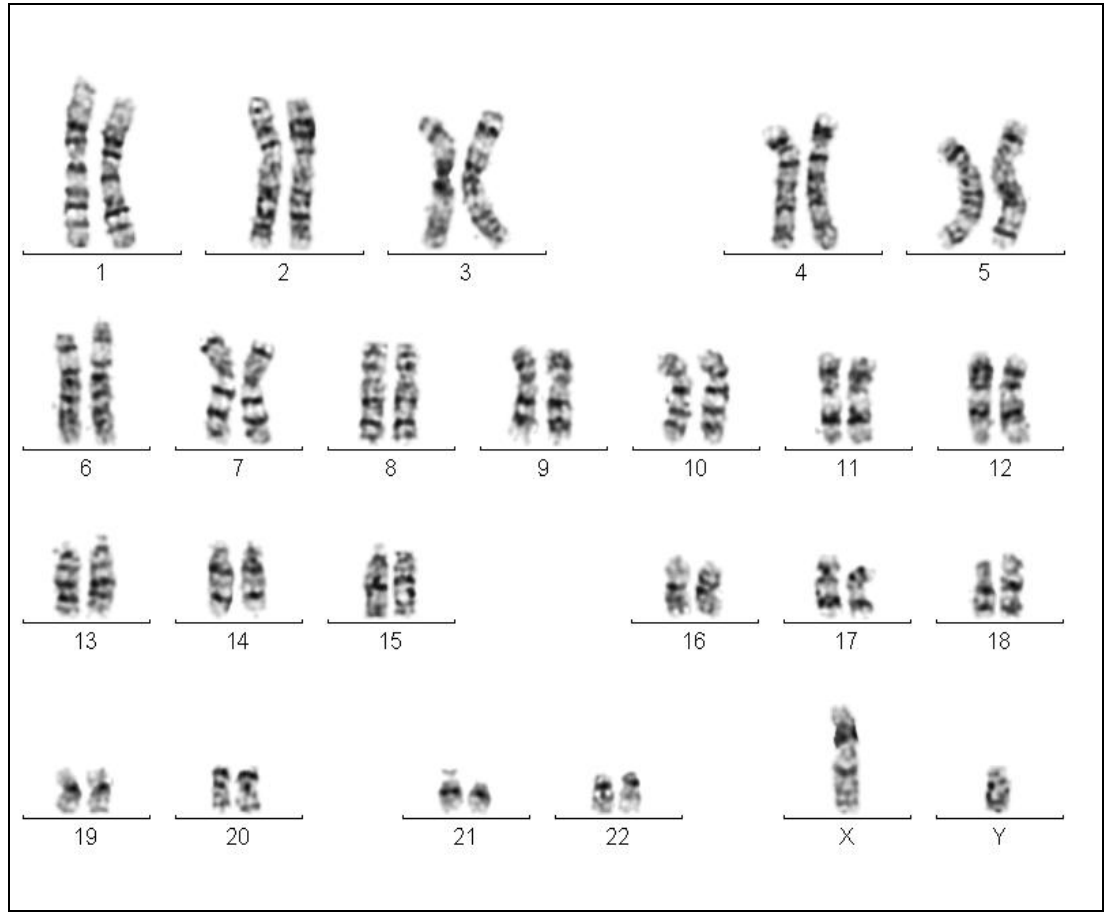

Fig 1 - GTG banded karyotype of Proband: 46,XY. 


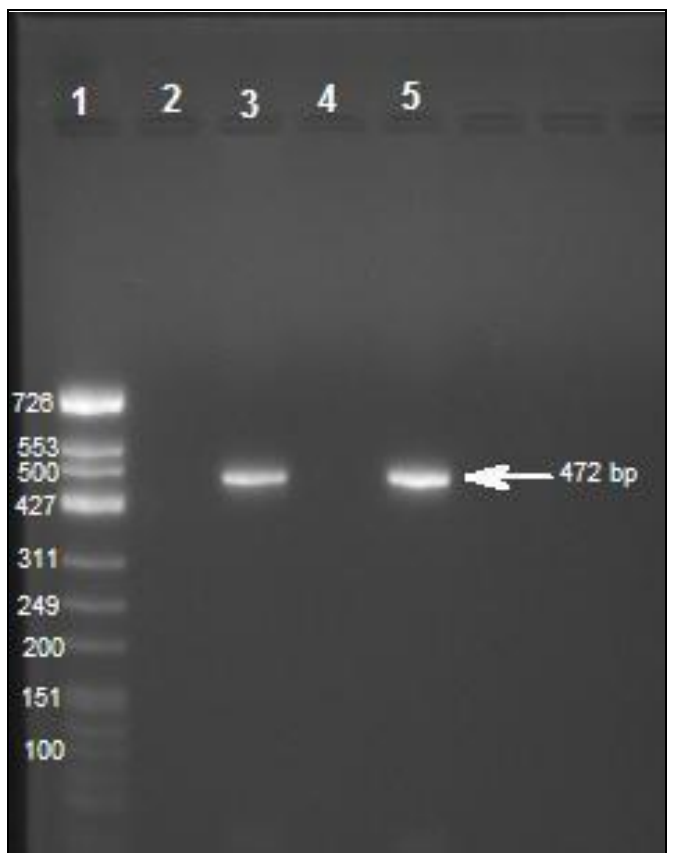

Fig 2- Presence of SRY gene, PCR analysis result (Lane1- Ladder, 2-Blank, 3- Proband, 4-female control, 5male control)

\section{DISCUSSION}

AIS, also known as testicular feminization syndrome or Morris syndrome, is a rare form of male pseudo-hermaphroditism, characterized by androgen insensitivity resulting from an absence or abnormal cytosol receptor for androgens. Thus, despite possessing a male karyotype (46, XY); phenotypically patient presents as an asymptomatic female usually discovered during perimenarchal stage when the individual fails to menstruate. The testis is often undescended and the ovaries, uterus, fallopian tubes, and upper third of the vagina are typically absent. Externally, the labia majora and minora are usually well formed [8]. The underlying cause is a mutation in the androgen receptor gene localized in the long arm of the X chromosome Xq11-12.2. Although up to $70 \%$ of androgen receptor mutations are $\mathrm{X}$-linked recessive, $30 \%$ of mutations can also be sporadic de novo mutations [9].

There is an inability of the androgen receptor to bind testosterone and $5 \alpha$-dihydrotestosterone. The undescended testes can undergo a malignant change, and the risk is estimated to be approximately $5 \%$. Most of these neoplasms are either germ cell tumors or sex cord stormal tumors. The risk of malignancy in AIS is considerably lower before puberty and occurs at a later age than with other intersex disorders. Typically, patients older than 30 year are at greatest risk and the risk of malignancy reaches up to $33 \%$ in patients above 50 years of age [10]. Removal of gonads is therefore recommended after puberty once final height and breast development have been achieved.

AIS is thought to be related to approximately 6-10\% of cases of primary amenorrhea [4]. Many girls with AIS are tall because the Y-chromosome carries genes for extra height [11]. Partial androgen insensitivity syndrome is a rare disorder. By definition PAIS is an intersex condition marked by genital ambiguity in any individual whose biology include an identifiable mixture of male and female characteristics, regardless of the appearance of the genitalia at birth.

PAIS like CAIS is transmitted as an X-linked trait and is related to the mutations in Androgen Receptor gene. Though more than $95 \%$ of individuals with CAIS show such mutations; these are seen in < $50 \%$ of PAIS patients. The laboratory findings required for diagnosis include $46 \mathrm{XY}$ karyotype; as well as evidence of normal or increased synthesis of testosterone (T) [3] ,but the testosterone level of the proband was low in our case.

Although mutation analysis has not been done in our case, most cases of CAIS are due to mutations in the AR gene [6]. Partial AIS is at least as common as CAIS and the affected 46,XY individuals are almost always infertile. Carrier females have a 50\% chance of transmitting the $A R$ gene mutation in each pregnancy. Carrier testing is available on a clinical basis once the $A R$ mutation has been identified in an affected family member [6]. 


\section{Management Of Existing Problem}

If AIS is suspected, it is of critical importance to find the rudimentary male gonads (by means of MRI, pelvic echography, laparoscopy) and surgically remove them to prevent the onset of malignancies (teratoblastoma, gonadoblastoma). Additional treatment includes vaginal dilatation to avoid dyspareunia. It is critical that health care providers understand the importance of properly diagnosing newborn manifesting ambiguous genitalia. Furthermore, a child with a pseudo hermaphrodite phenotype should always undergo adequate endocrine and genetic testing to reach a conclusive diagnosis before gender is assigned and surgical interventions are carried out. A trial of androgen pharmacotherapy may help improve virilization in infancy.

Those individuals with PAIS who are raised as males may undergo urologic surgery such as orchipexy and hypospadias repair. Those individuals with PAIS who are raised as females and who undergo gonadectomy after puberty may need combined estrogen and androgen replacement therapy. Systematic disclosure of the diagnosis of AIS is an empathic environment with both professional and family support, is encouraged [6].

\section{CONCLUSION}

The AIS is a rare X-linked recessive disorder of male pseudohermaphroditism with a male genotype and female external genitalia. The possibility of PAIS should be considered in any case of primary amenorrhea. Early diagnosis is important because the risk of malignancy in intra-abdominal testes is high. Patients with AIS need proper counseling and education according to their psychosexual make-up and socio-cultural factors.

\section{Acknowledgements}

The authors thank Mr. Abhimanyu Kumar and Mr. Sushil Singh of PreventiNe Life Care Pvt Ltd. for their involvement and support for the study.

\section{REFERENCES}

[1] X. Yuan, W. De-fen, L. Xiao-ying, Y. Jun and W. Wei, S578N mutation of the androgen receptor in an adolescent with complete androgen insensitivity syndrome, Chinese Medical Journal,123(11),2010, 1473-1476.

[2] A. Galani, S. Kitsiou-Tzeli, C. Sofokleous, E. Kanavakis, A. Kalpini-Mavrou, Androgen insensitivity syndrome: clinical features and molecular defects. Hormones (Athens),7, 2008, 217-229.

[3] S. Sharma, K. Singh, S. Dhar, Y. Gupta ,Partial Androgen Insensitivity Syndrome, JK Science, 12(1), 2010,41-43.

[4] B. Li, Q. Ding, X. Wan and X. Wang, Clinical and genetic characterization of complete androgen insensitivity syndrome in a Chinese family, Genetics and Molecular Research, 10 (2),2011,1022-1031.

[5] M. Oakes, A. Eyvazzadeh, E. Quint and Y. Smith, Complete androgen insensitivity syndrome-a review, Journal of Pediatric and Adolescent Gynecology,21, 2008, 305-310.

[6] B. Gottlieb, L. Beitel, M. Trifiro, Androgen Insensitivity Syndome, Gene Reviews. [Initial posting 24 March 1999 and Revised 24 May 2007]. Available from: http:// www.geneclinics.org/ profiles/androgen/detail.html.

[7] M. Felix, ISCN 2009: An International System for Human Cytogenetic Nomenclature ( Karger, Switzerland,2009).

[8] D. Rasalkar, B. Paunipagar, A. Ng, F. Lai, S. Bagaria , Intra-Abdominal Testicular Seminoma in a Woman with Testicular Feminization Syndrome. Case Reports in Radiology, 2011, Article ID 592124.

[9] N. Chauhan, Y. Sharma, S. Sharma, S. Sharma, Complete androgen insensitivity syndrome with microtia: a rare Presentation, Japanese Journal of Radiology, 29, 2011, 67-71.

[10] F. Zorlu, M. Cengiz, M. Gurkaynak, F. Yildiz, I. Atahan, Seminoma arising in androgen insensitivity syndrome (testicular feminization syndrome: A case report, Turkish Journal of Cancer, 31( 4), 2001,168-171.

[11] G. Wa r n e, Complete Androgen Insensitivity Syndrome (Printgraphics, Australia,1997). 